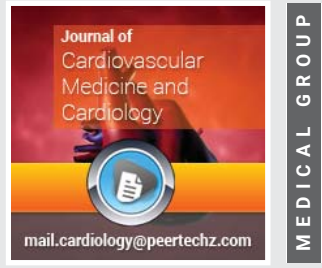

\title{
The COVID-19 pandemic is one of the components of the multicomponent pandemic of the 21st century
}

\author{
GM Bushmanova* and MV Supotnitsky \\ Institute of Molecular Pathology and Pathomorphology, FIC FTM. Novosibirsk, FSBI "27 Scientific \\ Center" of the Ministry of Defense of the Russian Federation, Moscow, Russia
}

Received: 19 May, 2020
Accepted: 27 May, 2020
Published: 28 May, 2020

*Corresponding author: GM Bushmanova, Institute of Molecular Pathology and Pathomorphology, FIC FTM. Novosibirsk, FSBI "27 Scientific Center" of the Ministry of Defense of the Russian Federation, Moscow, Russia, Tel: 8-913-770-15-43; E-mail: gbushmanova37@mail.ru

ORCID: https://orcid.org/0000-0002-5012-5608

https://www.peertechz.com

Check for updates
Let us try to look at the "new" problem by the example of two "linked" pandemics, which are prepared for the possibility of coexistence in the 21st century.

Today there can be no doubt the evidence of a significant age difference between the little-known COVID-19 that was born this year and that we have studied enough for a 40-year period (1980-2020), until the conscious medical community, primary chronic sepsis (P-ChS), associated with primary chronic septic endovasculitis, implemented in most cases in endocarditis (P-CSE) [1,2]

We are talking about pulmonary vasculitis as a particular manifestation of systemic vasculitis with a wide palette of clinical manifestations from minor and moderate to catastrophically malignant. The first and main active person is the initially highly virulent SARS-COV-2 virus found in patients.

A feature of the scenario is the fact that the battles of the disease unfold on the initially affected field in the conditions of a latent systemic non-specific endovasculitis accompanied by a high epidemic threshold. That is, the situation is characterized by the presence of a highly specific process against the background of sepsis, which is highly non-specific. In both cases, we are talking about vasculitis. CT-manifestations of pulmonary vasculitis and COVID-19 described in the literature are identical: pulmonary infiltrates in the form of "frosted glass".

The initial stage of COVID-19 is associated with a lesion of the cells of the alveolar epithelium virus. However, in situ replication of the virus with primary viremia occurring in this case is accompanied by damage to internal organs and systems. In this case, the vascular endothelium seems to be the inevitable next "target" for the defeat of the virus, which is realized in vasculitis.

The emerging clinical appearance of COVID-19, the real potentialities and consequences of which is still difficult to predict, cannot but be associated with one of the oldest diseases, sepsis, demonstrating on the example of patients all stages of its primary chronic variant from complete "clinical silence" to threatening urgent septic shock.

At first glance, there are at least two "linked" pandemics. One, suddenly bursting into the life of mankind, frightening with its so far known only immediate consequences and the other is hidden, the fact of existence of which we have not yet recognized by the representatives of the medical community, proved by us, and deserves serious discussion for a long time. Today it seems more formidable, due to the special severity of the life forecast for the species Homo sapiens as a whole.

How do these diseases differ from each other, in fact, having the same substrate of damage? We are talking about fibrosis, which affects the small vessels of the lungs with COVID-19 and universal vasculitis with damage not only to the endocardium, but also to the entire vascular system in primary chronic sepsis. Fibrosis of the alveoli in the first case basically seems to be the result of the implementation of the previously latent primary chronic vasculitis, the high activity of which is triggered by the initially highly virulent SARS-COV-2 (cytokine storm).

The fundamental difference between diseases is the different rate of formation of pathological substrate fibrosis: 
rapid, obstructing normal gas exchange at the alveoli level during COV infection, leading to the development of an urgent situation, and slowly progressing, gradual, as the results of our study showed with the initial latent pandemic of endovasculitis, gradually involving endocardial and endovascular structures, creating the conditions for the unitary genesis of the main acquired human diseases and the formation over time of a systemic organosclerosis-structural basis for the formation of organ acquired pathology and chronic heart failure [1,3-5].

In the situation with covid, the process of progressive vascular fibrosis in general, in the long run, even after the "cure" of the virus, is very real, only, perhaps, clinically more manifest its consequences can appear at the level of the pulmonary vessels. Demonstrating a pulmonary fibrosis clinic as a long-term consequence of COVID-19. The term of "remoteness" of this consequence will depend on many reasons and, first of all, on the achievement or insufficient achievement of the level of "cure" of the disease in each individual patient. The question of a complete cure for this virus remains open. A large number of "clinically silent" carriers of the virus cannot but alarm.

The prominent domestic virologist Academician V. Zuev and co-authors of an elegant experiment, which ended with the discovery of the ability of the influenza virus to cause a slow viral infection in the offspring of mammals as a result of intrauterine infection of the fetus of pregnant females, notes that latent viral infections form not only after the disease, $\mathrm{He}$ believes that the latent form of the infectious process is more common and develops as a result of immunization of the body with a live virus or through vertical transmission of the virus from mother to fetus [6].

Is it surprising that nature used a specific agent to uniquely bind COVID-19 as the only possible one to create a septic process, which is the expression of the highest nonspecific in nature? Will it not turn out in the end that the actual antigenic palette in the etiological structure of the disease and its pathogenesis is richer and SARS-COV-2 is not the only other pathogen possible? Perhaps the trigger role characteristic of the pathogen will inevitably gradually be limited by representatives of other taxa that inhabit the body, moreover, some of them are causally related to the initial, which appeared in the body long before COVID-19, latent pandemic of endogenous sepsis [1]?

The question rightfully arises: is an isolated viral infection entitled to exist in the presence of sepsis? Evolutionaryecological antagonism inherent in viruses and bacteria [7], probably cannot allow them to appear simultaneously in a generally consistent, genetically determined tandem. The active role of each of them, apparently, is determined by the existence of specific temporal circumstances and characteristics of diseases.

It is well known that you cannot try to treat both bacterial and viral infections. The fact that this is so is evidenced by practice. According to our observations, often the course of clinically effective antibiotic therapy ends after some time with the appearance of a mild herpetic rash or the appearance of "out of the blue", without visible provoking factors, SARS. At the same time, active drug suppression of the bacterial microbiota that lifted its head with the help of antibiotics provides the virus with a kind of carte blanche, allowing them to reactivate and remind themselves of themselves by awakening and leaving the reservation. As for the real representative of coronaviruses, the question of its complete elimination from the body is a matter that is being solved by time.

In a number of patients, the severity of the condition, associated with the progression of the disease, is accompanied by a descent of the viral infection from the level of the upper respiratory department to the lower and the development of pneumonitis first, according to academician A.G. Chuchalin, and then pneumonia. This can inevitably complicate the parasitocenosis and the etiological structure due to the addition of a bacterial, and not a secondary, hospital (the possibility of the latter is excluded) attachment to a viral infection, but a "homegrown" causally associated with P-cholesterol present in the body, which has not yet been taken into account by doctors due to the existing gap of ideas about the latter.

In the early stages, the activity of the bacterial microbiota was apparently suppressed by the actively replicating virus. But then, apparently, at the stage of the subsequent development of the disease, bacteria take the first, leading place, which determines the severity of the patient's condition, the damaging effect of which in the conditions of already existing septic endovasculitis acquires special severity. Moreover, it may not necessarily be pneumococci. The question of the existence of purely viral infections in humans, especially against the background of existing sepsis, seems debatable.

Is it possible to be completely sure of the exclusively "noncardial" genesis of pulmonary edema, since we are talking about the undeniably "septic portrait" of the disease with the accompanying hemostatic disorders like DIC, cytokine storm, oxidative stress, thrombophilia, thrombocytopenia, acute respiratory Di Stress Syndrome (ARDS) of an "increasing cascade of multiple organ failure" (SPON), Septic Shock (SS)? The results of an echocardiographic study with determination of the LV ejection fraction (EF) could answer this question. If this is so, then we can talk about a combination of respiratory failure, which has a structural basis, with acute LV-weakness, aggravating the severity of the patient's condition.

The global anthropological problem is directly related to the problem of anthropozoon infection, which has become the result of overcoming the species barrier. Many years have attracted the attention of A.V. Terletsky and L.G. Akhmerov. We are talking about one of its aspects - widespread in the Russian Federation (93\%) human invasion by intraerythrocytic representatives of Protozoa, malaria-like babesias of the family Babesiidae of the order Piroplasmida [8-10]. In 2007, in Moscow, at the V World Congress on Immunopathology and Allergy, our report was presented, "A New Clinical Phenomenon of the XX-XXI Centuries - Primary Chronic Septic Endocarditis and Blood-Parasitic Diseases", which met with great interest [11]. 
It is possible that the effect noted by Chinese scientists on chloroquine and other quinine derivatives in patients with COVID-19 may be required to suppress this latent protozoan invasion that occurs in these patients. You can verify this when conducting a blood test using the Romanovsky-Giemsa method in the modification of VA. Terletsky and L.G. Akhmerovoy. In this case, we are talking not only about babesias, but also rickettsia, as well as almost unchanged companions of babesia l-proteo-bacteria-bartonella.

As the results of our study showed, antiprotozoal therapy in combination with the main in patients with primary chronic septic endocarditis complicating sepsis is accompanied by a clear decrease in parasitemia by babesia and an often observed mild increase in the number of bartonella.

This suggests the possibility of evolutionary-ecological antagonism between babesia and bartonella, which is a bacterium taxon, and possibly requires the exclusion of simultaneous antibacterial and antiprotozoal therapy for patients with COVID-19. In addition, maybe from these positions it is worth considering a combination of antiviral and antiprotozoal therapy? This may allow a more correct assessment of the therapeutic possibilities of quinine preparations and drugs of a different orientation when they are simultaneously prescribed in the treatment of these patients, increasing the effect of therapy.

The anemia observed in patients with COVID-19 can be associated not only with a high degree of sepsis activity, but also with the presence of babesia in the red blood cells, using hemoglobin as a food nutrient. The question arises: How does the voice of a blood parasitic infection sound in this pandemic chorale today? What real contribution can it make and does it make? So far we can only speculate, but what apparently does not have to be doubted is that we are talking about a parallelgoing, secretly proceeding generalized invasion, of a pandemic level, causally obliged to a detachment of bloodsuckers, claiming their permanent representation in the human body and animals.

The urgency of the problem of blood parasitic infection is of particular importance in connection with the progressive invasion of ticks "spreading" across countries by humans in the Russian Federation. The existing ideas about the exceptional exoticism of babesiosis (pyraplasmosis) for the Russian Federation are a deep misconception, holding back indepth studies in this direction.

The above considerations regarding the possible treatment of patients with COVID-19. Suggest that it is necessary to observe a certain sequence in the use of basic drugs and their possible combinations. Antibacterial therapy must be carried out under the guise of antimycotics and probiotics.

Thus, everything turned out to be much more complicated than it seemed at first glance. The exclusivity of the situation, as it seems to us today, is that COVID-19 in its pure form, without $\mathrm{P}-\mathrm{XC}$ and does not exist. Representatives of the virusbacterial-protozoal microworld, who have their own interests and their own views on the nature of partnership with a person who is confused before an unexpected real threat to his life, are actively and confidently opposing a person. The observed picture is a reflection of the combination of pandemics of these two diseases, and not as a simple arithmetic addition, but as a complex integration of one with the other. We are aware that in reality it is not about 2 pandemics.

The past twentieth century was also unusually generous and presented to mankind, along with its greatest discovery of antibiotics, the ensuing pandemic of endogenous sepsis, about the possibility of which the outstanding infectious disease specialist A.F. Bilibin; pandemic associated with members of the Herpesviridae family (according to WHO since 1999); HIV / AIDS pandemic ... Today, viral hepatitis, with their threatening prospect, will eventually use all the letters of the alphabet, T-cell leukemia and possibly pandemics, not only causally associated with various representatives of the viralbacterial nature, to demonstrate their many real incarnations representatives of Protozoa.

Outstanding scientist academician V.P. Treasurers drew attention to a new phenomenon - endobiospheric epidemics in the human body, as an expression of microcoevolution $[12,13]$. It seems that one of the manifestations of this natural phenomenon at present is the pandemic of endogenous primary chronic sepsis. COVID-19, based on the data available today about it, has a different genesis. The list of pandemics promises to be continued. Nature cannot be indebted to the proud and crowned fruit of her own creation, which has not once crossed the line of the rational and permissible.

Taking into account the coexistence of a coronavirus pandemic with other pandemics of the present expands the possibilities for a deeper understanding of the chaos that occurs in the body, which is the result of the disintegration of processes characteristic of each pandemic, aggravating the already critical state of patients with coronavirus infection. Understanding and exploring this complex aspect of the pandemic problem can help optimize therapeutic approaches.

What awaits humanity of the planet after an imaginary victory over COVID-19? It should be understood that the present pandemic of endogenous sepsis, a priori programmed for a lifetime, will not leave a person a guarantee of a comfortable existence. Everything that will doze off or actively exist imperceptibly for a person will wake up, in reality, causing enormous damage to society. You need to be prepared for this. The prospect of the problem extends to infinity, for the rest of the life of successive generations who can survive under the conditions of this most difficult exo- and endoecological pressure, working to prematurely launch the implementation of the 2nd law of thermodynamics in the human body.

"Gentlemen, the last word for the microbe!" When was the great Louis Pasteur really right? When he said this phrase, or when, leaving the mortal world, he threw: "A microbe is nothing, an organism is everything!"

It seems (MV Supotnitsky) that there is reason to consider the real COVID-19 pandemic as one of the components of a multicomponent, pandemic non-cyclic infection process, a 
catastrophic natural phenomenon [14]. Mono-pandemics do not currently exist. Features of a real catastrophe of a planetary level put forward the requirements of urgent fundamental research with the mobilization of the intellectual potential of world scientific thought.

\section{References}

1. Bushmanova GM, Zorina IG, Terletsky AV, Akhmerova LT (2020) A new clinical phenomenon of the $\mathrm{XX}-\mathrm{XXI}$ centuries is primary chronic septic endocarditis as an argument against the diagnosis of rheumatism. Journal Therapy 2: 34-55

2. Bushmanova GM, Zorina IG, Nepomnyashchikh LM (2014) Primary chronic endocarditis - a new facet of the problem of sepsis and endocarditis. I Russian Congress with international participation. Proliferative syndrome in biology and medicine 91-97.

3. Bushmanova GM, Zorina IG, Ostankovich Ol (2013) From isolated parietal endocarditis to mitral stenosis - the path covered in 29 years. VIII National Congress of Therapists 53-54.

4. Bushmanova GM, Zorina IG, Ostankovich OI (2018) Primary chronic septic parietal endocarditis, as part of a long-term prospective observation of the patient, after 35 years. IV European Congress of Rheumatologists M Scientific and practical rheumatology 56: 24

5. Bushmanova GM, Zorina IG, Ostankovich OI (2018) History of progression of parietal primary chronic endocarditis, in the framework of prospective observation of the patient (1985-2018). VI Congress of Physicians of Siberia. Novosibirsk 49
6. Victor Zuev, The Many-Faced Virus. Secrets of hidden infections, Science and the world. M: AST-PRESS; 268.

7. Chereshnev VA, Morova AA, Ryamzina IN (2000) Biological laws and human vitality (Method of multifunctional restorative biotherapy). Russia-Czech Republic 168

8. Terletsky AV, Akhmerova LT (2005) Babesiosis (pyraplozmoz) - blood-borne disease of animals and humans. Hematology and transfusiology 50: 3.

9. Bushmanova GM, Terletsky AV, Akhmerova LG, Guseva IA (2008) Causa relationships of cardiac arrhythmias and primary chronic septic endocarditis associated with blood-parasitic infections. Allergology and immunology 8 : 259.

10. Terletsky AV, Akhmerova LG (2009) A method for the diagnosis of blood parasitic infections. Novosibirsk: LLC SHI-KON. patent No. 2362095

11. Bushmanova GM, Terletsky AV, Akhmerova LG (2007) Primary chronic septic endocarditis and blood parasite diseases. A new linical phenomenon of 2021 st centuries. Intrnational Proceedings of the V Europen Astma Congress and I World Congress on COPD. Moscow: Bologne MEDIMOND International Proceedings 59-62.

12. Treasurers VP, Nepomnyashchikh GI (2000) Thoughts on the problems of general pathology at the turn of the XXI century. Novosibirsk 47s

13. Kaznacheev VP, Dmitriev AN, Mingazov IF (2007) Civilization in conditions of growing energy intensity of natural processes of the earth, Novosibirsk: LLC Spk Dyunas 422.

14. Supotnitsky MV (2010) Microorganisms, toxins, epidemics. 3rd edition. M. "University book" 376

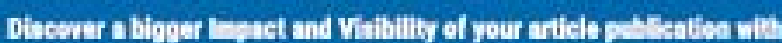
Peertechz Publications

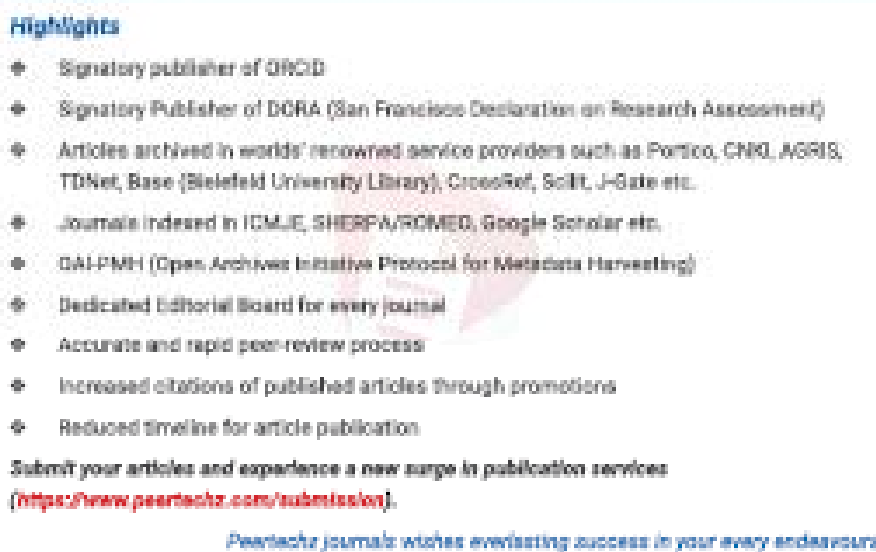

Copyright: (c) 2020 Bushmanova GM, et al. This is an open-access article distributed under the terms of the Creative Commons Attribution License, which permits unrestricted use, distribution, and reproduction in any medium, provided the original author and source are credited. 